\title{
An Efficient Method of Determining Diffusion Coefficients Using Eigenfunction Expansions $^{\dagger}$
}

\author{
K. Yip \\ Nuclear and Astrophysics Laboratory, University of Oxford, Keble Road, Oxford OX1 3RH, United Kingdom \\ K. Y. Tam ${ }^{\S}$ \\ Physical and Theoretical Chemistry Laboratory, University of Oxford, South Parks Road, \\ Oxford OX1 3QZ, United Kingdom \\ K. F. C. Yiu*, \\ Oxford University Computing Laboratory, Wolfson Building, Parks Road, \\ Oxford OX1 3QD, United Kingdom
}

Received June 28, $1996^{\otimes}$

\begin{abstract}
A method using eigenfunction expansions has been implemented to determine the diffusion coefficients of electroactive species. The method has been successfully applied to the process of single-electron oxidation of $N, N, N^{\prime}, N^{\prime}$-tetramethyl-p-phenylenediamine (TMPD). The results obtained by the method agree very well with those from the well established finite difference algorithm and are more reliable in general than the conventional Levich equation. The computer code developed is versatile and efficient; the method could be of wide application.
\end{abstract}

\section{INTRODUCTION}

Reaction rates and the mechanisms of many electrochemical processes are governed by the diffusion of reactants and product species. Knowledge of diffusion coefficients of the species involved is of utmost importance in the analysis and modeling of kinetic data. Electroanalytical techniques, such as chronopotentiometry, voltammerty, and polarography, provide simple but effective ways for evaluating diffusion coefficients of various electroactive species. ${ }^{1}$ However, the diffusion coefficients determined from these techniques have not been widely accepted, because the accuracy is limited by the validity of the equations describing the electrode currents. ${ }^{2}$ For instance, in dropping-mercury electrodes or rotating-disc electrodes, the relevant equations contain a number of approximations and assumptions. An alternative technique is to use the radiotracer method ${ }^{2}$ which can give very accurate measurements of diffusion coefficients, but this technique requires the use of specially labeled chemical elements which increases the cost significantly.

The channel electrode process is another useful technique to probe the kinetics and mechanisms of electrochemical reactions. ${ }^{3}$ A flow cell consisting of a rectangular duct and a working electrode is used. Solutions are passed into the cell under laminar flow condition. Since the hydrodynamics inside the cell is well defined, the electrode response as a function of mass transport can be calculated precisely and compared with the experimental values to gain mechanistic

\footnotetext{
* To whom all correspondence should be addressed.

Key words: diffusion coefficient, channel electrode, inverse problem, optimal control, eigenfunction expansion.

¥E-mail address: k.yip1@ physics.oxford.ac.uk.

§ Present address: Sirius Analytical Instruments Ltd., Riverside, Forest Row Business Park, Forest Row, East Sussex RH18 5DW, United Kingdom.

${ }^{\perp}$ Present address: Department of Engineering Science, University of Oxford, Parks Road, Oxford OX1 3PJ, United Kingdom.

${ }^{\otimes}$ Abstract published in Advance ACS Abstracts, February 15, 1997.
}

insight. In this manner, a variety of electrochemical reaction mechanisms have been resolved. ${ }^{4,5}$

In solving the convection-diffusion equation with a full laminar parabolic velocity profile for hydrodynamic electrode processes, Levich ${ }^{6}$ assumed a linear velocity profile to derive an approximate equation (the Levich equation) that yields the limiting current of the electrode as a simple function of the flow rate of the solution inside the electrode. The diffusion coefficient can be evaluated by fitting experimental data to the Levich equation. In this paper, a more reliable way of predicting the diffusion coefficients is proposed. The method is based on an inverse formulation, and the problem is solved by using an optimal control approach. A cost function is defined to be the mismatch between the experimental data and that calculated from the convection-diffusion equation (as defined below by (1.1) in section 2). The optimal diffusion coefficient is then extracted as that for which the cost function is minimized. Here, we solve this optimal control problem by using an eigenfunction expansion. We show that the optimal diffusion coefficient can be calculated by simply solving a nonlinear equation. The proposed method is demonstrated by application to the single-electron oxidation of $N, N, N^{\prime}, N^{\prime}$-tetramethyl-p-phenylenediamine (TMPD) and compared with the Levich equation procedure.

\section{THEORY}

Consider a laminar flow of a solution entering a channel electrode (see Figure 1) in which the diffusion in the $\mathrm{x}$ and $\mathrm{z}$ directions can be neglected. The governing convectiondiffusion equation for this channel electrode process is given by

$$
v(y) \frac{\partial g}{\partial x}=D \frac{\partial^{2} g}{\partial y^{2}}
$$




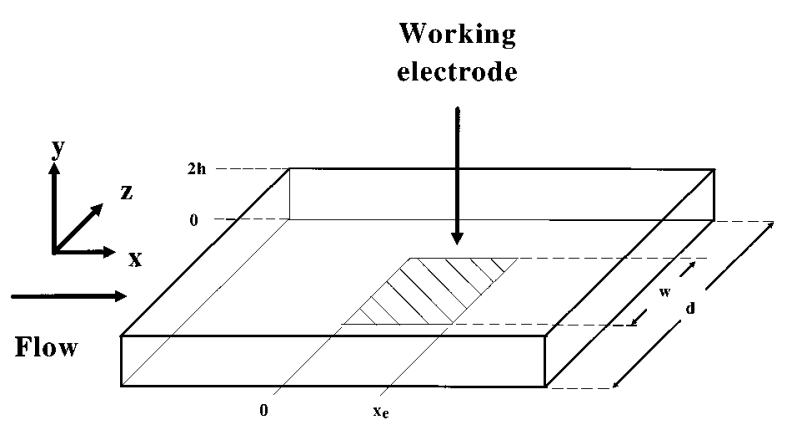

Figure 1. Schematic diagram of a flow cell in a channel electrode: see text for experimental details.

together with the boundary conditions defined in Figure 2, where $g$ is the concentration of the species, $D$ is the diffusion coefficient, and $v(y)$ is the laminar parabolic velocity profile given by

$$
v(y)=\frac{3 v_{f}}{4 h d}\left(1-\frac{(y-h)^{2}}{h^{2}}\right)
$$

where $v_{f}$ is the flow rate of the solution of interest in $\mathrm{cm}^{3}$ $\mathrm{s}^{-1}$, while $h$ and $d$ are defined in Figure 1. This equation can be simplified slightly using the transformation

$$
\bar{y}=\left(\frac{y-h}{h}\right), \quad \bar{y} \in[-1,1]
$$

and the initial and boundary value problem can be written as

$$
\begin{gathered}
v(y) h^{2} \frac{\partial g}{\partial x}=D \frac{\partial^{2} g}{\partial \bar{y}^{2}} \\
g(x,-1)=0, \quad \frac{\partial g}{\partial \bar{y}}(x, 1)=0, \quad g(0, \bar{y})=g_{0}
\end{gathered}
$$

In terms of $g$, the limiting current can be expressed as

$$
I=\left.\frac{w D F}{h} \int_{0}^{x_{e}} \frac{\partial g}{\partial \bar{y}}\right|_{\bar{y}=-1} \mathrm{~d} x
$$

where $F$ is Faraday's constant, while $w$ and $x_{e}$ are defined in Figure 1. Consequently, the inverse problem of finding $D$ can be formulated as

$$
\min _{D} E \equiv \sum_{j=1}^{m}\left(I_{j}-I_{\exp _{j}}\right)^{2}
$$

where the $\left\{I_{\exp _{j}}\right\}$ are the experimental limiting currents and $m$ is the number of data points. Because eq 1.4 can easily be solved by a two-dimensional finite difference grid algorithm, ${ }^{7}$ an obvious way of tackling eq 1.6 is to use a quasi-Newton optimization technique ${ }^{8}$ which can be summarized as follows:

(1) Choose an initial $D^{(0)}$.

(2) Solve eq 1.4 using $D^{(k)}$.

(3) Calculate $D^{(k+1)}$ using a quasi-Newton update.

However, this method requires solving eq 1.4 repeatedly for each iteration and is therefore expensive and inefficient.

An alternative approach is to make use of the fact that eq $1.4 \mathrm{a}$ is linear and homogeneous, so that variables can be separated by writing

$$
g(x, \bar{y})=X(x) Y(\bar{y})
$$

from which eq $1.4 \mathrm{a}$ becomes

$$
\frac{X^{\prime}(x)}{D X(x)}=\frac{Y^{\prime \prime}}{v(\bar{y}) h^{2} Y(\bar{y})}
$$

Using the independence of $x$ and $y$, each side of eq 1.8 must be a fixed constant, to be denoted $\lambda$; hence, we can write

$$
\begin{gathered}
X^{\prime}(x)+\lambda D X(x)=0 \\
Y^{\prime \prime}(\bar{y})+\lambda v(\bar{y}) h^{2} Y(\bar{y})=0
\end{gathered}
$$

Together with the boundary conditions

$$
Y(-1)=0 ; \quad \frac{\partial Y}{\partial \bar{y}}=0
$$

1.10 and 1.11 form a regular Sturm-Liouville problem ${ }^{9}$ which has an infinite number of eigenvalues:

$$
\lambda_{1}<\lambda_{2}<\lambda_{3}<\ldots ; \quad \lim _{n \rightarrow \infty} \lambda_{n}=\infty
$$

furthermore, each eigenvalue corresponds to an eigenfunction which is unique up to a normalization constant. These eigenfunctions will be denoted by $\left\{Y_{1}, Y_{2}, Y_{3}, \ldots\right\}$. Note that the $\lambda$ 's take positive values here because negative $\lambda$ 's would produce exponential increasing $X(x)$ which is nonphysical, while $\lambda=0$ yields the trivial solution $g \infty 0$. By using the principle of superposition, the general solution $g$ can be written as

$$
g(x, \bar{y})=\sum_{i=1}^{\infty} A_{i} \exp ^{-\lambda_{i} D x} Y_{i}(\bar{y})
$$

where the coefficients $\left\{A_{i}\right\}$ are to be calculated from the initial value using

$$
g_{0}=\sum_{i=1}^{\infty} A_{i} Y_{i}(\bar{y})
$$

Substituting eq 1.13 into eq 1.5 yields

$$
I=-\left.\left.\frac{w D F}{h} \sum_{i=1}^{\infty} \frac{A_{i}}{\lambda_{i} D} \exp ^{-\lambda_{i} D x}\right|_{0} ^{x e} \frac{\partial Y_{i}}{\partial \bar{y}}\right|_{\bar{y}=-1}
$$

On using this in eq 1.6, the required diffusion constant $D$, appears as an explicit argument of $E$; consequently, the necessary and sufficient conditions for a minimum are simply

$$
\frac{d E}{d D}=0, \quad \frac{d^{2} E}{d D^{2}}>0
$$

\section{EXPERIMENT}

In a test of this approach, the channel cell shown schematically in Figure 1 was a rectangular duct (about 4.5 $\mathrm{cm}$ long, $0.1 \mathrm{~cm}$ deep, and $0.6 \mathrm{~cm}$ wide) cut in a block of Black Derlin (Goodfellow Advanced Materials, Cambridge) and closed by a cover plate of the same material. A solution was made to flow through the channel via inlet and outlet teflon tubes which were attached to the ends of the cell along 


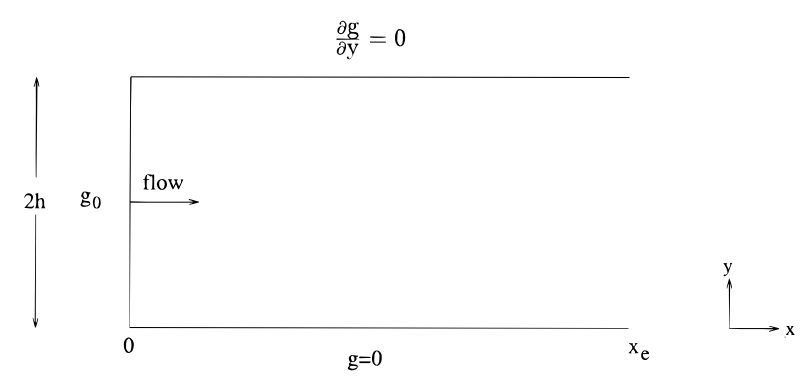

Figure 2. Representative boundary conditions in the formulation of the eigenfunction expansion method.

the $y$-direction. The working electrode was made of platinum (Goodfellow Advanced Materials, thickness $0.1 \mathrm{~mm}$, purity 99.95\%) with dimensions of approximately $4 \mathrm{~mm} \times 4 \mathrm{~mm}$. Contact with the electrode were maintained through a hole in the cover plate. The precise dimensions of the electrode were measured to a precision of $\pm 0.002 \mathrm{~cm}$ by using a travelling microscope. Typical values obtained were $x_{e}=$ $0.394 \mathrm{~cm}, w=0.402 \mathrm{~cm}$ and $d=0.601 \mathrm{~cm}$ (see Figure 1). The depth of the cell $(2 h$, in Figure 1$)$ was determined accurately by using a depth gauge (Mitutoyo, Japan) to within $\pm 0.001 \mathrm{~cm}$. In our case, $2 h$ was found to be $0.0819 \mathrm{~cm}$.

The flow velocity was adjusted by linking the solution exit to a precalibrated capillary tube. A saturated calomel reference electrode (SCE) was placed on the upstream side of the cell, while a platinum counter electrode was situated downstream from the cell. All electrochemical measurements were carried out at $25{ }^{\circ} \mathrm{C}$ using a scan generator and a potentiostat (Oxford Electrode Ltd, UK) in conjunction with an Hewlett Packard 7035B X-Y recorder. The TMPD (98\%, Aldrich) was used as received. The supporting electrolyte was prepared from reagent-grade potassium chloride (Aldrich). Solutions used were made from deionized water of resistivity greater than $10^{7} \Omega-\mathrm{cm}$ and degassed thoroughly with nitrogen prior to use.

We elected to study this system because TMPD is wellknown to be oxidized reversibly to the radical cation at a half-wave potential of $0.015 \mathrm{~V}$ vs. SCE. ${ }^{10}$ In the present study, electrochemical experiments were performed using $2.12 \mathrm{mM}$ TMPD in a $0.20 \mathrm{M} \mathrm{KCl}$ solution. We varied the flow rate of the solution while scanning the electrode potential of the working electrode from $-0.2 \mathrm{~V}$ to $+0.2 \mathrm{~V}$ at $10 \mathrm{mV} \mathrm{s}^{-1}$ corresponding to the transport-limited oxidation of TMPD. A half-wave potential of $0.01 \mathrm{~V}$ (vs SCE) was found and was observed to be essentially constant and independent of the solution flow rate which is consistent with the literature. ${ }^{10}$ This suggests that the ohmic drop between the working and reference electrode is negligible. ${ }^{11} \mathrm{We}$ observed that all voltammetric waves were of smooth sigmoidal shape and satisfied the Tomes criterion of reversibility. ${ }^{12}$ Note that an overpotential of more than $200 \mathrm{mV}$ was maintained for every measurement to ensure that the current was limited by mass transfer. Hence it is not unreasonable to assume that adsorption of TMPD or the radical cation on the working electrode surface is negligible $(<1 \%)$. Under these conditions, the current values obtained should directly reflect the transport limited currents of the oxidation process (although a significant adsorption on the electrode would degrade the accuracy). It must also be emphasized, however, that the quality of the electrochemical data will influence the accuracy of the diffusion coefficient as deduced in the next section.

\section{RESULTS AND DISCUSSION}

In practice, the Sturm-Liouville problem

$$
\begin{gathered}
Y^{\prime \prime}(\bar{y})+\bar{\lambda} Y(\bar{y})=0 \\
Y(-1)=0, \quad \frac{\partial Y}{\partial \bar{y}}=0
\end{gathered}
$$

is solved using subroutines developed by NAG Ltd. ${ }^{13}$

A finite number of eigenvalues and eigenfunctions are used so that eq 1.14 is approximated by the finite series

$$
g_{0}=\sum_{i=1}^{n} \tilde{A}_{i} Y_{i}(\bar{y})
$$

There are two different ways of calculating the $\left\{\tilde{A}_{i}\right\}$. The first approach is to make use of the fact that the $\left\{Y_{i}(\bar{y})\right\}$ form an orthogonal basis with respect to the weight function (1 $-\bar{y}^{2}$ ) on the interval $-1<\bar{y}<1$, so that one has

$\tilde{A}_{i}=\int_{-1}^{1}\left(1-\bar{y}^{2}\right) Y_{i}(\bar{y}) g_{0} d \bar{y} / \int_{-1}^{1}\left(1-\bar{y}^{2}\right) Y_{i}^{2}(\bar{y}) d \bar{y}$,

$$
i \in[1, n]
$$

Another way is to employ all the eigenfunctions in calculating each $A_{i}$ by forming the matrix equation

$$
M \mathbf{a}=\mathbf{g}
$$

where

$$
\mathbf{a}=\left(\tilde{A}_{1}, \ldots, \tilde{A}_{n}\right)^{T}
$$

and

$$
g_{i}=\int_{-1}^{1} Y_{i}(\bar{y}) g_{0} d \bar{y}, \quad M_{i, j}=\int_{-1}^{1} Y_{i}(\bar{y}) Y_{j}(\bar{y}) d \bar{y}
$$

It turns out that eq 2.3 is more versatile because the addition of extra eigenfunctions does not affect the previously calculated coefficients; however, eq 2.5 is numerically more stable and eq 2.2 converges more rapidly numercially using fewer eigenfunctions. After finding the coefficients $\left\{\bar{\lambda}_{i}\right\}$, $\left\{\tilde{A}_{i}\right\}$, and $\left\{(\partial Y(-1) / \partial \bar{y})_{i}\right\}$ they are stored in an archive file for future retrieval.

Here, we emphasize that even if some of the experimental parameters such as $x_{e}, w, d$, and $2 h$ are changed, exactly the same set of eigenfunctions and eigenvalues can be used. This is because the eigenvalues and eigenfunctions are found without invoking any of these parameters. In fact, the most time-consuming process is finding the eigenvalues and eigenfunctions which are sought by using the algorithm described in ref 13. Once this has been done, it takes virtually no time to determine the diffusion coefficient from experimental data.

For comparison purposes, we consider the Levich equation $^{3}$ in which the parabolic velocity profile eq 1.2 is simplified to

$$
v(y) \simeq 6 \frac{v_{f}}{4 h d}\left(1-\frac{y}{h}\right)
$$

Note that the approximation of replacing the parabolic velocity profile by a linear function is satisfactory only when the flow is dominated by forced convection in the laminar regime. The analytic solution when using eq 2.6 is given 


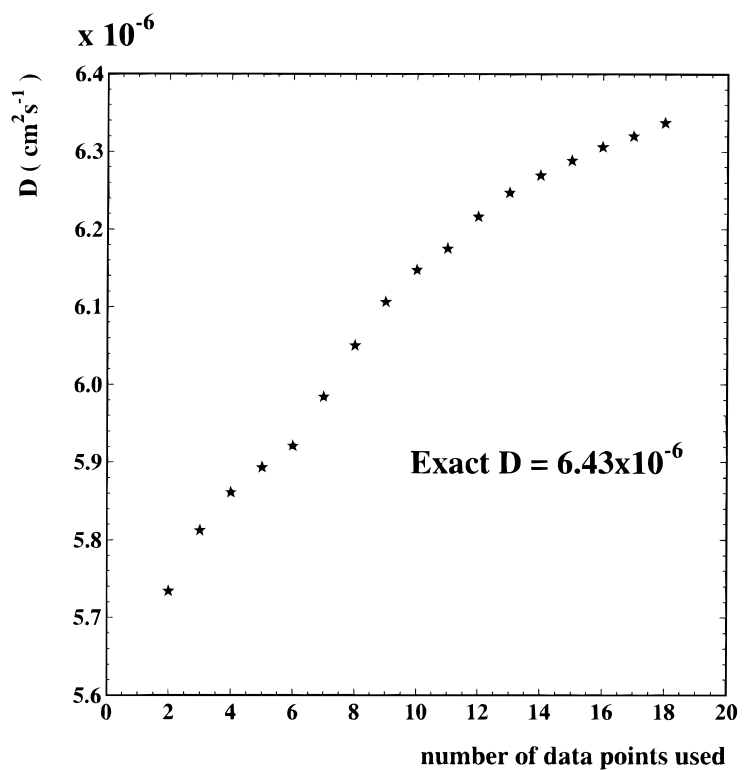

Figure 3. Diffusion coefficients calculated from the Levich equation as a function of the number of data points used.

$\mathrm{by}^{3}$

$$
I=0.925 n_{e} F w g_{0}\left(\frac{v_{f} D^{2} x_{e}^{2}}{h^{2} d}\right)^{1 / 3}
$$

where $n_{e}$ is the number of electrons transferred. Clearly, the limiting current $I$ is a linear function of $v_{f}^{1 / 3}$; hence, a linear regression analysis of these two variables allows $D$ to be deduced from the slope of the $I-v_{f}^{1 / 3}$ plot.

First, we illustrate a deficiency of the Levich equation approach. A hypothetical diffusion coefficient equal to 6.43 $\times 10^{-6} \mathrm{~cm}^{2} \mathrm{~s}^{-1}$ was assumed, and the finite difference algorithm with $1000 \times 1000$ mesh points was used to calculate $I$ in eq 1.5 with 18 different flow rates. The result from the Levich equation analysis is shown in Figure 3. Clearly, the predicted diffusion coefficients are very sensitive to the number of data points used. The Levich equation method is particularly inaccurate in the slow flow-rate region where a linear regression analysis is apparently not sufficient.

Secondly, we demonstrate the convergence of the eigenfunction expansion method in computing eq 1.5. The same diffusion coefficient as in the previous example was used; the results are compared with those found from the finite difference algorithm in Figure 4. Clearly, the method converges nicely and agrees well with the finite difference algorithm. More importantly, note that the method of eigenfunction expansions only took a trivial time (about 0.4 s of CPU time on a Digital Unix workstation) to calculate all the $I$ 's when the set of eigenvalues and eigenfunctions had been previously stored.

We have also compared the diffusion coefficients calculated by three different methods, namely the Levich equation, the finite difference quasi-Newton algorithm, and the eigenfunction expansion method. The experimental results are displayed in Table 1 for nine different flow rates. When

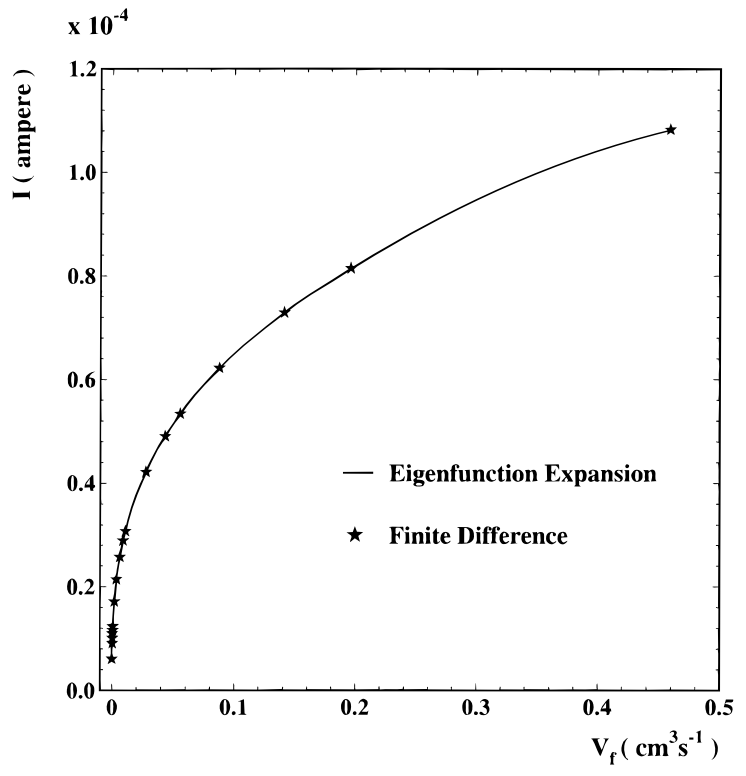

Figure 4. Limiting currents $(I)$ as a function of the flow rates $\left(v_{f}\right)$ evaluated by the finite difference algorithm and eigenfunction expansion method respectively.

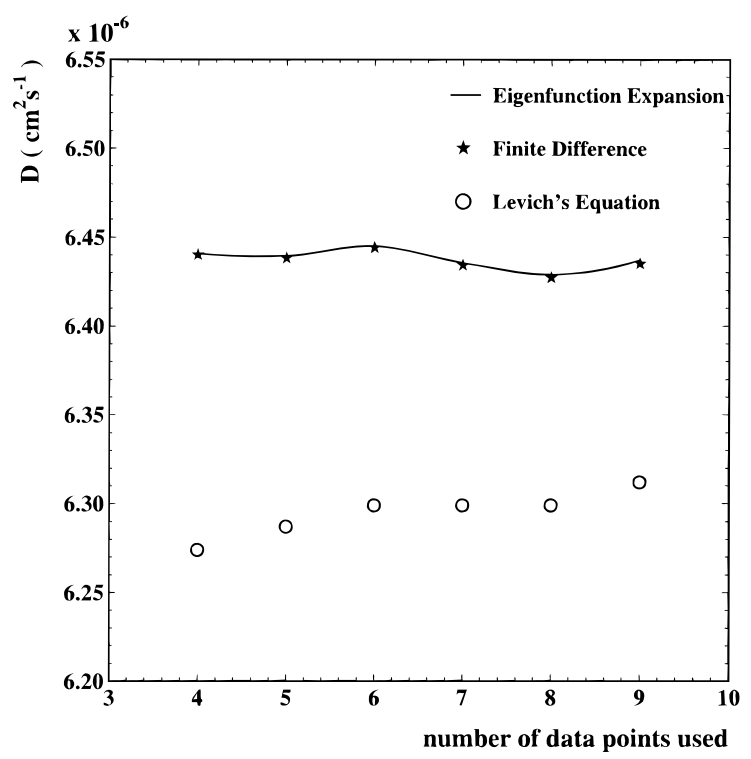

Figure 5. Comparison among three different methods: Levich equation, finite difference algorithm, and eigenfunction expansions.

using the eigenfunction expansion method, the optimizing equation $d E / d D=0$ was solved by applying Newton's method. The results are portrayed in Figure 5. We see that the Levich equation approach underestimates the diffusion coefficients systematically owing to its approximate assumption. By contrast the other two methods agree very well. Although the accuracy for these two methods is the same, their efficiencies differ greatly. The finite difference quasiNewton algorithm took about $60 \mathrm{~s}$ of CPU time to calculate each diffusion coefficient, while the eigenfunction expansion took virtually nothing to finish the same task $(0.4 \mathrm{~s}$ of CPU time on a Digital Unix workstation) once all the required eigenvalues and eigenfunctions had been stored. Using the

Table 1. Experimental Data Obtained by the Channel Electrode Technique ${ }^{a}$

\begin{tabular}{|c|c|c|c|c|c|c|c|c|c|}
\hline$v_{f}\left(10^{-3} \mathrm{~cm}^{3} \mathrm{~s}^{-1}\right)$ & 6.45 & 9.11 & 10.9 & 19.6 & 27.7 & 32.4 & 43.3 & 52.0 & 55.7 \\
\hline$I\left(10^{-5}\right.$ ampere $)$ & 2.62 & 2.88 & 3.08 & 3.74 & 4.22 & 4.46 & 4.89 & 5.20 & 5.36 \\
\hline
\end{tabular}




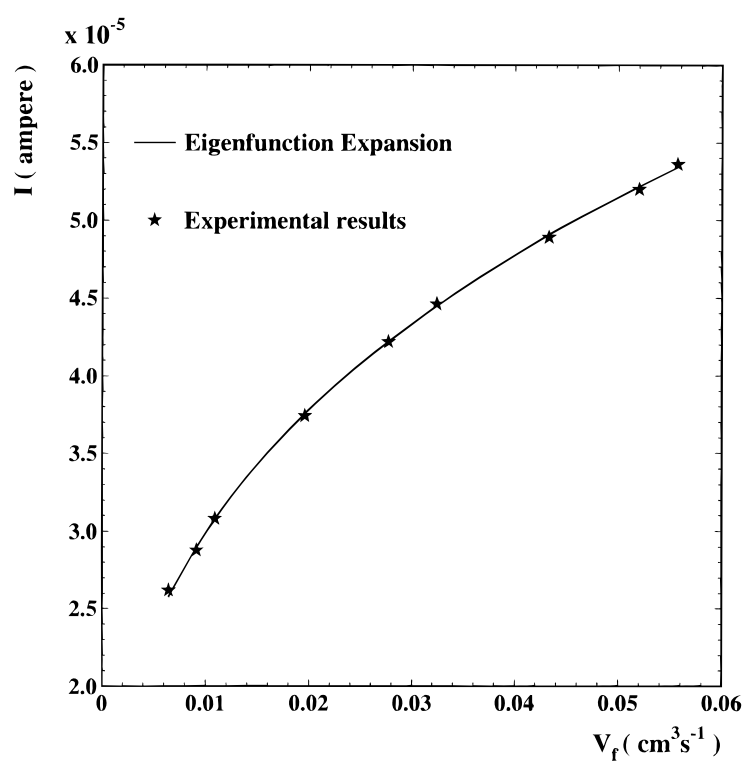

Figure 6. Comparison between the limiting currents evaluated from the eigenfunction expansion method and the experimental data: see text for experimental details.
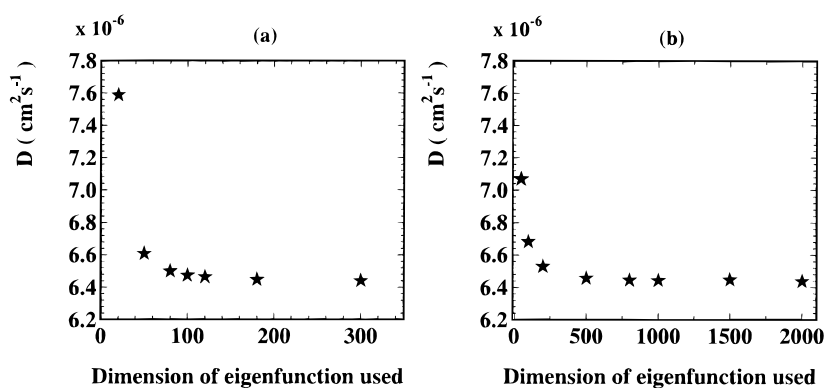

Figure 7. (a) Convergence in the calculation of the diffusion coefficients using the method based on eq 2.3. (b) Convergence in the calculation of the diffusion coefficients using the method based on eq 2.5.

diffusion coefficient extracted from all the nine data points by the method of eigenfunction expansion, the limiting currents (which are used to define the cost function in the optimisation process) were calculated and compared to the experimental values: see Figure 6.

Finally, the convergence rates of the two different integration methods eqs 2.3 and 2.5 has also been studied. All nine data points in Table 1 were used, and the results are shown in Figure 7 (parts a and b). We see that when eq 2.5 was used, the results converge much faster. The only disadvantage of using eq 2.5 is that the matrix equation eq 2.4 needs to be resolved whenever an extra eigenfunction is added. This increases the cost and complexity of the method. Consequently, it is generally preferable to use eq 2.3 because of its versatility in spite of the slower convergence.

\section{CONCLUSIONS}

An efficient method based on eigenfunction expansions has been developed to solve the convection-diffusion equa- tion for a channel electrode system. With this method, one needs to calculate the necessary eigenvalues and eigenfunctions only once: they can then be used to solve the mass transport equation for any cell geometry and flow rate of the solution. We have demonstrated that the optimal diffusion coefficient of the relevant electroactive species can be predicted easily by solving a simple nonlinear equation. The technique has been successfully applied to calculate the diffusion coefficient of TMPD in aqueous solution. Compared to the conventional approach using Levich's equation, our method not only provides a more reliable calculation of the diffusion coefficient but also has a much higher efficiency in terms of computational time than the finite difference quasi-Newton algorithm. Potentially, the eigenfunction expansion technique can be extended to model the computational intensive multispecies transport equations of electrochemical interests.

Program and data files are available from one of the authors, K. Yip upon request.

\section{ACKNOWLEDGMENT}

We would like to thank Professor R. G. Compton for providing the necessary equipment for all electrochemical measurements. We would also like to thank Dr. F. Marken for helpful discussion and the referee for various comments.

\section{REFERENCES AND NOTES}

(1) Adams, R. N. Electrochemistry at Solid Electrodes; Marcel Dekker: New York, 1969.

(2) Mills, R.; Lobo, V. M. M. Self-diffusion in Electrolyte Solutions; Elsevier: Amsterdam, 1989.

(3) Unwin, P. R.; Compton, R. G. Comprehensive Chemical Kinetics; Elsevier: Amsterdam, 1989; Vol. 29, Chapter 6.

(4) Waller, A. M.; Compton, R. G. Comprehensive Chemical Kinetics; Elsevier: Amsterdam, 1989; Vol. 29, Chapter 7.

(5) Compton, R. G.; Dryfe, R. W. A. Progress Reaction Kinetics; Elsevier: Amsterdam, 1995; Vol. 20.

(6) Levich, V. G. Physicochemical Hydrodynamics; Prentice-Hall: Englewood Cliffs, NJ, 1962.

(7) Pilkington, M. G.; Compton, R. G.; Stearn, G. M. Mass transport in channel electrodes. J. Chem. Soc., Faraday Trans. 1 1988, 84(6), 2155-2171.

(8) Fletcher, R. Practical Methods of Optimization; John Wiley: New York, 1987.

(9) Pruess, S.; Fulton, C. T. Mathematical software for Sturm-Liouville problems. ACM Transactions on Mathematical Software 1993, 19(3), 360-376.

(10) Philip, R. H. J. Electroanal. Chem. 1970, 27, 369-373.

(11) Coles, B. A.; Compton, R. G.; Spackman, R. A. Electroanalysis 1993, 5, 41-46.

(12) Bard, A. J.; Faulkner, L. R. Electrochemical Methods; John Wiley: New York, 1980.

(13) Marletta, M.; Pryce, J. D. Automatic solution of Sturm-Liouville problems using the Pruess method. J. Computational Appl. Mathematics 1992, 39, 57-78.

CI9604652 